\title{
MedienPädagogik
}

Zeitschrift für Theorie und Praxis der Medienbildung

Themenheft Nr. 37: Medienpädagogik als Schlüsseldisziplin in einer mediatisierten Welt. Perspektiven aus Theorie, Empirie und Praxis Herausgegeben von Henrike Friedrichs-Liesenkötter, Lara Gerhardts, Anna-Maria Kamin und Sonja Kröger

\section{Kooperation als Strategie - Einführung von E-Assessments an Hochschulen}

\section{Herausforderungen und Chancen in der Medienpraxis}

Marcel Graf-Schlattmann, Gudrun Oevel und Melanie Wilde

\section{Zusammenfassung}

Dieser Beitrag legt am Beispiel des von Prof. Dr. Dorothee M. Meister initiierten Projektes «E-Assessment NRW» dar, welche weitreichenden Herausforderungen es bei der Einführung von E-Assessments gibt. Dabei werden drei Handlungsfelder - Technik, Didaktik und Recht - bei der Einführung, Implementierung und Umsetzung von E-Assessments betrachtet und herausgestellt, welche Chancen eine kooperative Zusammenarbeit unter Berücksichtigung hochschulinterner Prozesse bietet und welche Konsequenzen sich daraus für die Medienpraxis an Hochschulen ergeben. Der Fokus des Beitrags liegt auf der kooperativen Zusammenarbeit im Bereich von Weiterbildungs- und Vernetzungsformaten. 


\title{
Cooperation as a strategy - Implementing E-Assesments at universities. Challenges and chances in media practice
}

\begin{abstract}
The paper "cooperation as a strategy - implementing e-assessments at universities》 shows, on the example of the "E-Assessment NRW» project initiated by Prof. Dr. Dorothee M. Meister, the challenges in the introduction of e-assessments at universities. In regarding to the three dimensions of -technology, didactics and law-we developed an approach for a cooperative basis in account to organisational structures at universities and their consequences for media practice. The paper focusses on the field of training and networking formats in higher education.
\end{abstract}

\section{Veränderungen durch die Einführung von E-Assessments}

Der Wandel an Hochschulen von klassischen, analogen Prüfungen zu elektronisch unterstützten Prüfungsformaten, den sogenannten E-Assessments, ist keineswegs trivial, sondern eine voraussetzungsvolle Entwicklung. Bestehende Grundannahmen zum Thema (digitales) Prüfen müssen überdacht und neu geschaffen werden um Herausforderungen und Potenziale transparent und nutzbar zu machen.

Bevor wir uns jedoch dem eigentlichen Thema dieses Beitrags, der Kooperation als Strategie für Einführungs-, Implementierungs- und Umsetzungsprozesse von E-Assessments an Hochschulen widmen können, braucht es ein Begriffsverständnis von E-Assessments. ${ }^{1}$

Unter E-Assessments verstehen wir elektronische Prüfungen, die mit digitaler Unterstützung angeboten bzw. durchgeführt werden. Diese können diagnostisch, formativ oder summativ erfolgen, wobei sich die Anforderungen an den Einführungsprozess sowie die Möglichkeiten des Einsatzes von E-Assessments zwischen den einzelnen Typen unterscheiden (siehe Meister und Oevel 2017; Schmees und Horn 2014). Zum besseren

1 Der Begriff der Strategie wird an dieser Stelle weitgehend vorausgesetzt. Wir verstehen unter Strategie im Hochschulwesen einen sich stetig weiterentwickelnden Prozess, der sowohl top-down als auch bottom-up erfolgt. Siehe weiterführend: Graf-Schlattmann u.a. (2018) 
Verständnis erläutern wir daher an dieser Stelle in kurzer Form drei Assessmentformen sowie die damit verbundenen Veränderungen.

Diagnostische E-Assessments dienen dazu, die Ausgangslage im Lernprozess zu ermitteln. Der bekannteste Anwendungsfall ist der Einstufungstest zur Ermittlung des Sprachlevels. Dabei liegt der Schwerpunkt auf der Verbindung von didaktischen Aspekten und möglicher Software bzw. Tools für E-Assessments. Je nach Einsatzszenario können auch Fragen der Infrastruktur - sprich Räume und Hardware - relevant sein.

Bei formativen E-Assessments - einer prozessbegleitenden Betrachtung des Lernstands - nehmen Infrastrukturen eine eher untergeordnete Rolle ein, da der Einsatz weitgehend losgelöst von Räumen und Hardware erfolgen kann. Auswirkungen sind hier vor allem bei der Verbindung von Software und Didaktik zu erkennen.

Summative E-Assessments sind elektronische Prüfungen zum Abschluss einer Lerneinheit - bspw. in Form einer Klausur zum Ende des Semesters. Diese Prüfungsform ist mit der Frage nach der Rechtssicherheit verbunden. Da die Prüfungen in den meisten Fällen benotet sind stellen sie eine sogenannte berufsbezogene Prüfung dar, die Auswirkungen auf das weitere Studium sowie das zukünftige Arbeitsleben der Prüflinge haben. Aus diesem Grunde müssen sie strengen Rechtskriterien genügen, die auch bei analogen Prüfungen zu berücksichtigen sind. Siehe dazu weiterführend: Forgó, Graupe, und Pfeiffenbring (2016).

\section{Das Projekt E-Assessment NRW}

Ausgehend von den Beobachtungen zu Herausforderungen und Potenzialen, die mit der Einführung von E-Assessments einhergehen widmete sich Prof. Dr. Dorothee M. Meister in der damaligen Position als Vizepräsidentin für Studium und Lehre der Universität Paderborn diesem Thema. Im Jahr 2014 initiierte sie gemeinsam mit den Prorektorinnen und Prorektoren sowie den Vizepräsidentinnen und Vizepräsidenten für Studium und Lehre der Hochschulen in NRW das Projekt E-Assessment NRW, welches vom damaligen Ministerium für Innovation, Wissenschaft und Forschung (heute Ministerium für Kultur und Wissenschaft) des Landes NordrheinWestfalen für vier Jahre finanziert wurde. 
Das Verbundprojekt zwischen den Universitäten Duisburg-Essen, Wuppertal und Paderborn sowie den Hochschulen Ostwestfalen-Lippe und Niederrhein hatte zum Ziel, den Herausforderungen bei der Einführung von E-Assessments an Hochschulen in NRW durch Kooperation und gemeinsame Anstrengungen zu begegnen. Der vorliegende Beitrag fokussiert auf die Projektergebnisse ${ }^{2}$ von E-Assessment NRW vor der Fragestellung, wo lohnt sich Kooperation bei Projekten zur Digitalen Bildung an Hochschulen.

Ausgangspunkt des Verbundprojekts E-Assessment NRW waren Online-Befragungen unter der Leitung von Prof. Dr. Dorothee M. Meister an den Universitäten und Fachhochschulen in NRW zum Stellenwert elektronischer Assessments an Hochschulen, den förderlichen und hemmenden Bedingungen sowie dem Status Quo des Einsatzes. Die Befragungsergebnisse stellten Bedarfe in den Themenbereichen von technischen, didaktischen und rechtlichen Fragstellungen für die Einführung und Umsetzung von E-Assessments heraus. Basierend auf den Befragungsergebnissen wurden innerhalb des Projekts E-Assessment NRW drei Schwerpunkte gebildet: rechtliche Verfahrensweisen, Beratungs- und Infrastrukturen sowie Kooperationspotentiale und Verbundstrukturen.

Dabei wurden die Reduktion von Unsicherheiten beim Einsatz von EAssessments, das Sichtbarmachen und Vernetzen der Akteure, der Abbau von Hürden durch Praxisbeispiele, Gutachten, Handreichungen, und die Förderung der Akzeptanz für das Thema E-Assessments in der Hochschullehre als konkrete Ziele des Projekts festgelegt.

Der Fokus dieses Beitrags liegt auf der kooperativen Arbeit beim Lösen der Herausforderungen, wobei hochschulinterne Prozesse stets mitgedacht werden. Dieser Schwerpunkt ergibt sich daraus, dass u.E. den Herausforderungen sowohl auf didaktischer und technischer als auch auf rechtlicher Ebene durch einen kooperativen Zugang begegnet werden kann. Eine vermehrte und intensivierte Kooperation erscheint demnach als eine umfassende Strategie, die alle drei Ebenen gleichermassen berücksichtigt.

2 Die Ergebnisse des Projekts befinden sich in der Abschlussdokumentation des Projekts. Meister und Oevel (2017): «E-Assessments in der Hochschulpraxis. Empfehlungen zur Verankerung von E-Assessments in NRW.» Online verfügbar: http://www.eassessmentnrw.de/fileadmin/eassessmentnrw/pdf/E-Assessment_in_der_Hochschulpraxis.pdf 
Hierfür werden zunächst die sich stellenden Herausforderungen bei Einführungs-, Implementierungs- und Umsetzungsprozessen am Fall der E-Assessments dargestellt und mit der kooperativen Zusammenarbeit eine denkbare Form der Lösung aufbereitet In den folgenden Abschnitten richtet sich der Blick auf den typischen Modus der kooperativen Zusammenarbeit in Form von Weiterbildungs- und Vernetzungsformaten. Anschliessend wenden wir den Blick auf die drei identifizierten Handlungsfelder und stellen die Besonderheiten für die technischen, didaktischen und rechtlichen Facetten im Einführungsprozess von E-Assessments heraus. Abschliessend widmen wir uns im Fazit den sich daraus ergebenden Konsequenzen für die Hochschulpraxis.

\section{Herausforderungen und Chancen bei der Einführung von E-Assessments}

Das Projekt E-Assessment NRW identifizierte in Befragungen und während der Projektarbeit verschiedene Herausforderungen und Hemmnisse bei der Einführung von E-Assessments. Einerseits birgt der mit der Einführung von E-Assessments verbundene Veränderungsprozess Möglichkeiten zur Ausgestaltung, andererseits sind mit den didaktischen, technischen und rechtlichen Veränderungen auch Herausforderungen bei der Einführung und Umsetzung digitaler Prüfungsformate an Hochschulen verbunden. Diese Herausforderungen finden sich zu Teilen auch in anderen Veränderungsprozessen. So sind bspw. neue Herangehensweise in der Lehre fast immer mit einem Anpassungsbedarf in der Didaktik verbunden. Der Wechsel von analogen zu digitalen Prüfungsformen stellt jedoch einen deutlichen Bruch zum Bestehenden dar, sodass bspw. die bisherigen Formen zur Sicherstellung der Authentizität und Integrität nicht genutzt werden können und Lösungen neu entwickelt werden müssen.

Neben rechtlichen Fragen der Authentizität und Integrität im Bereich des summativen digitalen Prüfens sind insbesondere auch Fragestellungen zu mediendidaktischen Konzepten beim Einsatz von E-Assessments sowie technische Anforderungen an die Infrastruktur entscheidend. 
Typische praktische Beispiele hierfür sind die Fragen wer bei einer automatisierten Prüfung der eigentliche Prüfer ist oder welche Massnahmen ergriffen werden müssen um zu beweisen, dass die Prüfung wirklich von dem Studierenden abgelegt wurde - und nicht von anderen Personen - und diese nicht im Nachhinein verändert wurde. Während man bei einer analogen, schriftlichen Prüfung die Authentizität und Integrität der Prüfungsleistung mithilfe einer Ausweiskontrolle zu Beginn und einer Unterschrift zum Abschluss der Prüfung sicherstellen kann, sind diese Möglichkeiten bei einer digitalen Prüfung in dieser Form nicht gegeben. Bei summativen E-Assessments muss zusätzlich eine weitgehende Rechtssicherheit in allen Aspekten der Prüfung - Prüfungsvorbereitung, -durchführung, -bewertung sowie -archivierung - gewährleistet werden. Darüber hinaus sind auch technische Anforderungen an die Infrastruktur, u.a. im Bereich der Hard- und Software sowie didaktische Aspekte des Prüfens im Hinblick auf die Erreichung von Lernzielen zu beachten.

Diesen Herausforderungen kann auf unterschiedlicher Weise erfolgsversprechend begegnet werden. So kann differenziert werden, ob die Herausforderungen, innerhalb der eigenen Hochschule oder auf kooperativem Weg gelöst werden sollten.

Während das Schaffen einer geeigneten Infrastruktur für elektronische, summative Prüfungen noch von den einzelnen Hochschulen alleine bewältigt werden kann, gibt es eine Vielzahl von Themen und Herausforderungen, die nur auf kooperativer Ebene gelöst werden können, wie im Folgenden dargelegt wird.

Das Thema E-Assessment stellt, in der Breite unterschiedlicher Themen und Prozesse in den Hochschulen, nur einen kleinen Bereich der Hochschulentwicklung dar und kann oftmals nicht von einer einzelnen Hochschule personell und organisational ausreichend abgedeckt werden.

Aus diesem Grund bietet es sich gerade für vermeintlich kleine Themen an, einen kooperativen Ansatz zu wählen. Denn der Prozess zur Einführung, Umsetzung und Etablierung von E-Assessments an Hochschulen ist nicht mit dem erfolgreichen Einsatz elektronischer Assessments in einigen Lehrveranstaltungen oder dem Schaffen und zur Verfügung stellen der benötigten Infrastruktur seitens der Hochschule beendet. Er beginnt oftmals gerade erst damit, dass die Grundlagen geschaffen und der Einsatz 
in einigen Pilotprojekten bzw. Lehrveranstaltungen erprobt wurde. Sobald dies erreicht ist, gilt es, den Prozess in die Tiefe und Breite zu bringen und stetig weiterzuentwickeln. Geeignete Mittel hierzu liegen bspw. in den Bereichen von Weiterbildung und Vernetzung.

Weiterbildungen finden in NRW im Grossteil der Fälle hochschulextern und im Zusammenschluss statt. Die Themen von Weiterbildung und Vernetzung sind daher eng miteinander verbunden, und können nicht getrennt voneinander betrachtet werden. Ebenso sind die Möglichkeiten von Weiterbildung und Vernetzung nicht auf ein bestimmtes Themenfeld, beispielsweise didaktische Angebote, begrenzt, sondern lassen sich auch für technische sowie rechtliche Herausforderungen nutzen.

Im weiteren Verlauf werden die Bedarfe zuerst allgemein dargestellt und anschliessend auf die Besonderheiten in den Bereichen von Technik, Didaktik und Recht eingegangen. Letztendlich sind sowohl Technik, Didaktik als auch insbesondere Rechtsfragen kooperativ zu lösen.

Ein bedeutendes Hemmnis für den Einsatz von E-Assessments stellen rechtliche Unsicherheiten dar. Da die potentiellen Konsequenzen eines nicht rechtskonformen Einsatzes sehr weitreichend sind, müssen vor Einführung und Einsatz von E-Assessments die rechtlichen Fragestellungen beantwortet sein. Wird der Einsatz von E-Assessments fokussiert, sind unterschiedliche Akteursgruppen einer Hochschule, wie Lehrende, Mitarbeiterinnen und Mitarbeiter aus Technik und Verwaltung, der Hochschulleitung oder auch der Justiziariate, mit unterschiedlichen Herausforderungen und damit einhergehend auch unterschiedlichen Klärungs- und Informationsbedarfen zu berücksichtigen. Damit sind wiederum konkrete Weiterbildungs- und Vernetzungsbedarfe verbunden, da die unterschiedlichen Akteursgruppen zwar heterogene Anforderungen und Interessen haben, letztlich jedoch alle Hochschulen vor denselben Herausforderungen stehen. Auf diesem Wege können relevante Synergieeffekte ermöglicht werden.

Eine weitere Facette ist die Sichtbarkeit der Akteure. Denn durch eine erhöhte Sichtbarkeit von Hochschulen und Einzelpersonen, die sich mit dem Thema E-Assessments allgemein und den unterschiedlichen Assessmenttypen konkret befassen, steigt sowohl der Grad der Vernetzung als auch das Potenzial von Weiterbildungen. 
Wichtig ist es dabei neben den unterschiedlichen Interessen und Anforderungen, auch das unterschiedliche Vorwissen bei den verschiedenen Akteurinnen und Akteure zu beachten. Denn ebenso wie in der Lehre allgemein hat man es beim Thema von E-Assessments mit Novizen, Fortgeschrittenen und Expertinnen und Experten zu tun.

\section{Modus der Kooperation: Weiterbildung und Vernetzung}

Die Heterogenität der verschiedenen Akteurinnen und Akteure sowie deren unterschiedliches Vorwissen schafft Chancen für eine kooperative Zusammenarbeit. Durch Austauschformate kommt es beispielsweise zu einem gegenseitigen Anreichern der Kenntnis- und Umsetzungsstände und förderliche sowie hemmende Faktoren und Entwicklungen werden sichtbar. Denn, der aktuell verbreitete und bevorzugte Modus der Kooperation ist die Weiterbildung und Vernetzung. Die Weiterbildungs- und Vernetzungsaktivitäten dienen der Sensibilisierung und Aktivierung auf der Ebene von Novizen sowie der Unterstützung von Erfahrungsaustausch und dem Kennenlernen neuer Trends und Möglichkeiten für Fortgeschrittene sowie Expertinnen und Experten.

Der Austausch kann dabei noch eine weitere Funktion einnehmen. Die unterschiedlichen Wissens- und Kenntnisstände bei den Akteuren sind oftmals auch mit den unterschiedlichen Fortschritten im Umsetzungs- bzw. Einführungsprozess verbunden. Vernetzung kann auch dafür genutzt werden, von den Erfahrungen anderer zu lernen und eventuelle Fehler nicht zu reproduzieren. Auf ein solches, häufig auftretendes, Problem im Umsetzungsprozess von E-Assessments an Hochschulen, aber auch anderer Veränderungsprojekte, sei an dieser Stelle explizit hingewiesen: Die Projektergebnisse von E-Assessment NRW haben gezeigt, dass es für den Erfolg entscheidend ist, alle beteiligten Akteursgruppen möglichst frühzeitig einzubinden. Diese Erkenntnis ist auch in anderen Projekten und Empfehlungen zu Veränderungsprojekten zu finden, dennoch kommt es häufig vor, dass relevante Akteurinnen und Akteure spät eingeschaltet werden und so der Erfolg des Prozesses gefährdet wird.

Weiterbildung und Vernetzung muss nicht an ein bestimmtes Treffen an einem Ort gebunden sein, sondern kann ebenso digital erfolgen. Die 
Vorteile liegen dabei auf der Hand: Die zeitliche und räumliche Trennung der Weiterbildungsaktivitäten - beispielsweise in Form eines Webinars - ermöglicht eine höhere Flexibilität und sorgt somit potenziell für mehr Gehör und Interesse.

Des Weiteren können solche Formate zusätzlich die Nachhaltigkeit von Weiterbildung und Vernetzung fördern und zu Synergieeffekten führen. Denn Weiterbildung findet nicht nur in grossen hochschulübergreifenden Zusammenschlüssen statt, sondern natürlich auch hochschulintern. Insbesondere die hochschuldidaktischen Weiterbildungen sind an dieser Stelle zu nennen, denn aufgrund der hohen Anzahl von Lehrenden finden diese meist selbstorganisiert an der jeweiligen Hochschule statt.

Auch die technische Weiterbildung findet zu gewissen Teilen hochschulintern statt, manchmal auch gekoppelt mit der didaktischen Weiterbildung. Das rechtliche Themengebiet verfügt hingegen meist nicht über ausreichend viele und unterschiedliche Akteure, dass es spezifische, hochschulinterne Weiterbildungsmassnahmen rechtfertigt.

Durch den Einsatz von Jour-Fixe-Formaten oder Webinaren können einerseits die internen Weiterbildungen geschickt mit kurzweiligen Formaten angereichert werden und andererseits auch neue Zielgruppen erreicht werden, die zu einer Tagung oder einem klassischen Netzwerktreffen beispielsweise nicht erscheinen würden und nur über Umwege erreicht werden könnten.

Des Weiteren können solche Formate das Risiko einer zunehmenden Homogenisierung der Akteurinnen und Akteure mindern und die Reflexion der eigenen Arbeit fördern, da regelmässig neue Personen angesprochen werden können.

Ein weiterer Vorteil von digitalen Weiterbildungs- und Vernetzungsangeboten liegt darin, dass sich die Verbreitung der Inhalte potentiell multipliziert und die Effekte eine grössere Reichweite erhalten können. Teilnehmende von Weiterbildungs- und Vernetzungsveranstaltungen bilden sich nicht nur für sich weiter, sondern auch als Vertreter und Vertreterinnen der jeweiligen Hochschule. Damit einher geht die Erwartung, dass sie die neu erworbenen Kenntnisse über Trends und Möglichkeiten von den hochschulübergreifenden Veranstaltungen in die eigenen Hochschulen übertragen und als Multiplikatoren tätig sind. Insbesondere bei einem 
neueren Thema, wie es E-Assessments zu Projektbeginn waren, ist dieser Faktor von grosser Bedeutung.

Nachdem nun der Modus und die Vorteile von Weiterbildungs- und Vernetzungsaktivitäten allgemein beleuchtet wurde, soll im Folgenden der Fokus auf die Spezifika und Möglichkeiten der einzelnen Aspekte von Technik, Didaktik und Recht gelegt werden.

\section{Besonderheiten technischer Fragestellungen: Marketplace, Aus- tausch}

Die technischen Fragestellungen sind noch am ehesten von den einzelnen Hochschulen selber zu lösen. Aus der Frage, welches technische Equipment (Hardware) zur Verfügung steht, ergeben sich bestimmte Anforderungen an die zu benutzenden Räume und an die Organisation (inkl. Software) der Prüfung vor Ort.

Dies gilt natürlich auch andersherum: Stehen nur Räumlichkeiten mit gewissen Eigenschaften zur Verfügung, muss die Technik daran angepasst und die Organisation auf diese Gegebenheiten abgestimmt werden. Die Bereiche von Software, Technik und Organisation sind also eng miteinander verbunden.

Bevor dies jedoch möglich ist, müssen an jeder Hochschule zunächst die Grundlagen geschaffen und gemeinsam mit rechtlichen Fragestellungen ausgelotet werden.

Hier bieten sich Möglichkeiten eines hochschulübergreifenden Austausches zu Open-Source-Lösungen an, da mit dem Einsatz elektronischer Prüfungen der Stellenwert der Technik steigt. Des Weiteren ist jeder Veränderungsprozess auch mit potentiellen Fehlentwicklungen verbunden, die im Austausch ausgeräumt werden können. Da der relevante Unterschied zu klassischen Assessments in der elektronischen Durchführungsform liegt, ist fast jeder Austausch über E-Assessments selbstverständlich mit der technischen Seite verbunden. Dementsprechend sind Netzwerke im Bereich der E-Assessments - beispielsweise die Digitale Hochschule NRW (DH NRW), die Arbeitsgruppe Online-Klausuren der Arbeitsgemeinschaft der Medieneinrichtungen an Hochschulen E.V. (AMH OnlineKlausuren) oder das E-Prüfungs-Symposium (ePS) - immer auch mit einer technischen Weiterbildung verbunden. 


\section{Besonderheiten didaktischer Fragestellungen: Beratung als Teil didaktischer Weiterbildungsnetzwerke}

Im Bereich der Hochschuldidaktik liegt ein zusätzliches Potenzial kooperativer Problemlösungen darin, die Weiterbildung und Vernetzung um den Aspekt der Beratung, im Sinne eines weiteren unterstützenden Faktors für eine erfolgsversprechende Einführung von E-Assessments, zu erweitern.

Unterstützung und Beratung zu technischen oder rechtlichen Neuerungen im Bereich von E-Assessments ist ebenfalls zentral für eine erfolgreiche Einführung. Im Vergleich zu diesen beiden Bereichen ist der Effekt in der didaktischen Weiterbildung jedoch weitaus stärker ausgeprägt, da diese ohne miteinbeziehender Beratung weit unter ihren Möglichkeiten bliebe. Daher liegen die vorrangigen Beratungsbedarfe im Bereich der Hochschuldidaktik allgemein sowie speziell in der (E-)Prüfungsdidaktik.

Die Beratung ist als relevanter ergänzender Teil der Weiterbildung zu verstehen, bei dem stärker auf die individuellen Bedarfe eingegangen werden kann. Dies kann in Form einer Einzel- oder auch in Form der kollegialen Beratung erfolgen. Die technische Beratung zu E-Assessments findet meist an den bereits etablierten E-Learning-Beratungsstellen an den Hochschulen statt.

Im Optimalfall ist an dieser Stelle auch der didaktische Gesichtspunkt integriert. Eine spezielle E-Assessment-Beratungsstelle ist hochschulübergreifend in NRW bisher nicht vorhanden.

Im Bereich der rechtlichen Aspekte von E-Assessments ist insbesondere auch an Beratungen und Austausch unter den Verantwortlichen im Justiziariat zu denken.

Eine ausgebaute Service- und Beratungsstruktur war ein zentrales Anliegen der Hochschulvertretung bei den Ausgangsbefragungen des Projekts E-Assessment NRW. Sowohl hinsichtlich der didaktischen als auch der technischen Aspekte von E-Assessments wurde angemerkt, dass eine solche Struktur den Einsatz erleichtern und fördern könnte. Dies hat sich auch im Projektverlauf als erfolgskritischer Faktor erwiesen. Für eine erfolgsversprechende Implementierung von E-Assessments sollten also die bestehenden Unterstützungsangebote innerhalb der Hochschulen weiter ausgebaut und zusätzlich die Verbindung von technischen, rechtlichen und didaktischen Weiterbildungs- und Beratungsangeboten auch in den 
existierenden unterschiedlichen hochschulübergreifenden Netzwerken (z.B. Hochschuldidaktische Netzwerke, Justiziare, Datenschutzbeauftragte) gefördert werden. Da die (hochschul-) didaktische Beratung im Feld der E-Assessments in NRW noch nicht so systematisch etabliert ist, können durch Austausch, Weiterbildung und Vernetzung Impulse für eine Entwicklung der Beratungsangebote geliefert werden. Auch Weiterentwicklungen hinsichtlich kompetenzorientierten E-Assessments sollten stärker in den Blickwinkel genommen werden.

Auf Ebene didaktischer Gesichtspunkte können bei der Planung von E-Assessments Lernzieltaxonomien unterstützend genutzt werden. Dabei werden die im Constructive Alignment formulierten Lernziele kategorisiert, was sowohl die Formulierung von Lernzielen unterstützt als auch den Transfer von Lehrinhalten und Assessments erleichtert, da man sich bei Erstellung der Assessmentfragen an den für die Lernziele festgelegten Taxonomiestufen orientieren kann. Ein weiterer bedeutender Vorteil kann darin gesehen werden, dass so das hochgradig individuelle Lehren, Lernen und Prüfen dokumentiert werden kann und dies Grundlage einer fortlaufenden didaktischen Qualitätssicherung von durchgeführten EAssessments sein kann. Auch die individuellen Beratungsanforderungen können dadurch sinnvoll unterstützt werden.

\section{Besonderheiten rechtlicher Fragstellungen: Schaffung von Grundlagen durch ein Rechtsgutachten}

Rechtliche Fragestellungen und Unsicherheiten bilden ein Kernthema des Einsatzes elektronischer Prüfungen. Grundlagen sind zum einen verfassungsrechtlicher und zum anderen datenschutzrechtlicher Natur. Auf Basis dieser Grundlagen ergibt sich die Herausforderung, Prüfungsordnungen und -systeme so zu gestalten, dass elektronische Prüfungen mit dem grösstmöglichen Mass an rechtlicher Absicherung durchgeführt werden können.

Im diesem Bereich lag im Jahr 2014 ein ausgeprägter Bedarf vor. So gaben die befragten Hochschulangehörigen in der Ausgangsbefragung an, dass es keine Rechtssicherheit bzgl. elektronischer Prüfungsformen gäbe bzw. rechtliche Bedenken gegen die Einführung von E-Assessments an ihren Hochschulen bestünden. 
In dieser Feststellung ist das wohl bedeutendste Hemmnis zur Etablierung und Implementierung von E-Assessments zu sehen. Da die rechtlichen Anforderungen alle Hochschulen gleichermassen betreffen und dabei zu umfangreich sind, als dass sie neben den laufenden Aufgaben gelöst werden könnten, sind die rechtlichen Fragestellungen ein klassischer Fall für kooperative Zusammenarbeit. Die Auflösung rechtlicher Hemmnisse durch das Projekt E-Assessment NRW wird daher hier exemplarisch für die Potentiale kooperativer Arbeit angeführt.

Von Projektseite wurde den rechtlichen Hemmnissen durch ein beauftragtes Gutachten zu den rechtlichen Aspekten von E-Assessments an Hochschulen (siehe Forgó, Graupe, und Pfeiffenbring 2016) und daraus abgeleiteten Handlungsempfehlungen begegnet. So mussten die Justiziariate der einzelnen Hochschulen sich nicht mit den alle Hochschulen betreffenden rechtlichen Anforderungen befassen, sondern konnten die gebündelte Expertise nutzen und sich auf das juristische Gutachten berufen.

Das Gutachten befasste sich mit den verfassungsrechtlichen Grundlagen zum Einsatz von E-Assessments und den daraus abzuleitenden Anforderungen an die Prüfungsordnungen, Prüfungssysteme und den Ablauf einer elektronischen Prüfung (Vorbereitungs-, Durchführungs-, Abschluss- und Archivierungsphase). Neben den verfassungsrechtlichen Grundlagen sind noch weitere Anforderungen zu beachten. Durch die 2018 in Kraft getretene DGSVO (Datenschutzgrundverordnung) (Europäische Union 2016) ergeben sich auch datenschutzrechtliche Veränderungen, die die Hochschulen vor erneute rechtliche Herausforderungen beim Einsatz von E-Assessments, aber auch elektronischer Learning- und Campus-Managementsysteme, stellen.

Anders als im Bereich der didaktischen Weiterbildungs- und Vernetzungsangebote wurden keine neuen Initiativen gegründet, sondern bewusst bestehende Netzwerke - insbesondere das Treffen der Justiziare und Justiziarinnen der Hochschulen in NRW - genutzt. Dabei handelt es sich weniger um ein Weiterbildungs-, sondern vielmehr um ein Austauschund Vernetzungsforum. Die Vernetzung als regelmässige Aktivität in einer community of practice ist darüber hinaus auch auf Ebene der Hochschulleitungen und den dazugehörigen Netzwerken aus dem Prorektorat sowie dem Vizepräsidium von grosser Bedeutung. 


\section{Fazit und kritische Reflexion}

Am Beispiel des von Prof. Dr. Dorothee M. Meister initiierten Projektes E-Assessment NRW wurde in diesem Beitrag herausgestellt, welche weitreichenden Herausforderungen es bei der Einführung von E-Assessments gibt und welche Chancen eine kooperative Zusammenarbeit bietet.

Es zeigt sich, dass der Einsatz von E-Assessments in den Hochschulen vor Ort stattfindet. Die Grundlagen dafür sowie die erfolgversprechende Ausgestaltung wird am ehesten kooperativ und im Austausch zwischen den Hochschulen geschaffen. Denn der Bereich elektronischer Assessments ist so komplex, dass die Einführung einzelne Hochschulen überfordern kann. Dies zeigt sich sowohl bei dem Thema der technischen Grundlagen als auch insbesondere in der rechtlichen Thematik, die eine grundlegende Forschung zu Beginn des Projekts E-Assessment NRW bedurfte, sowie im Bereich der didaktischen Konzepte.

Des Weiteren konnte anhand des Themas der Weiterbildung und Vernetzung ein zielführender Modus der Kooperation aufgezeigt werden, der in allen Facetten der Einführung (technisch, didaktisch, rechtlich) eingesetzt werden kann.

Im Bereich der didaktischen Weiterbildung lässt sich darüber hinaus auch der Aufbau von Beratungsmöglichkeiten als Kooperationsthema benennen. Die Beratung findet innerhalb der Hochschulen statt. Bei EAssessments handelt es sich aber um einen Bereich unter vielen und das Thema ist zu spezifisch, als dass es in jeder Hochschule passende Experten und Expertinnen gibt. Hierin liessen sich ebenso die Potentiale einer kooperativen Zusammenarbeit aufzeigen. Zum Abschluss sollen kurz kritische Stimmen berücksichtigt und die, vermutlich bedeutendste, Gefahr der kooperativen Arbeit reflektiert werden. Denn neben den vielfältigen Vorteilen von Weiterbildungs- und Vernetzungsaktivitäten für eine erfolgsversprechende und insbesondere nachhaltige Einführung von E-Assessments, konnten im Projekt E-Assessment NRW auch Herausforderungen identifiziert werden. Sie liegen darin, dass jede Kooperation Overhead (Zeit- und Personalressourcen) erzeugt und die Ziele der Vernetzung über das förderliche Mass erreicht werden können und dadurch negative Effekte auf den Prozess haben könnten. 
Ein denkbares Szenario wäre, dass sich beispielsweise die Vernetzungsaktivität von den eigentlichen Zielen löst. Die Vernetzung fände dann als Selbstzweck des Vernetzungswillens statt und nicht mehr, um die potenziellen Vorteile zu nutzen.

Ein zweites Szenario der Übererreichung von Vernetzungsaktivitäten liegt in der Anzahl von Netzwerken und Austauschformaten.

Das Thema der E-Assessments ist facettenreich und besteht aus vielen unterschiedlichen Aspekten. Würde jeder denkbare Aspekt beispielsweise in einem eigenen spezifischen Netzwerk behandelt, würde man schnell vor lauter Netzwerken das eigentliche Thema nicht mehr sehen und diejenigen, die über eine grosse und breite Expertise verfügen, wären gänzlich damit beschäftigt, diese auf einer Vielzahl von Netzwerktreffen zu präsentieren.

Beide Szenarien haben gemein, dass der Grenznutzen des Netzwerkens erreicht ist und die Aktivitäten schädlich statt förderlich wären.

Diese Gefahr ist jedoch der Vernetzung immanent und betrifft nicht das Thema E-Assessment allein. Da die Thematik der E-Assessments jedoch relativ neu auf dem (hochschul-)bildungspolitischen Tableau ist, ist die Netzwerkstruktur und -dichte noch im Fluss und kann dementsprechend leicht über das förderliche Mass hinausgehend erreicht werden. Bei aller Förderung von Kooperation sollte daher auch stets die Frage nach der Sinnhaftigkeit der Anzahl von Netzwerken gestellt werden. 


\section{Literatur}

Europäische Union. 2016. «Verordnung (EU) 2016/679 des Europäischen Parlaments und des Rates vom 27. April 2016 zum Schutz natürlicher Personen bei der Verarbeitung personenbezogener Daten, zum freien Datenverkehr und zur Aufhebung der Richtlinie 95/46/EG». https://eur-lex.europa.eu/legal-content/DE/TXT/PDF/?uri=CELEX\%3A32016Ro679\&from=de.

Forgó, Nikolaus, Simon Graupe, und Julia Pfeiffenbring. 2016. «Rechtliche Aspekte von E-Assessments an Hochschulen. Gutachten im Auftrag des Verbundprojektes E-Assessment NRW». Universität Duisburg-Essen. http://duepublico.uni-duisburg-essen.de/servlets/DocumentServlet?id=42871.

Graf-Schlattmann, Marcel, Dorothee M. Meister, Gudrun Oevel, und Melanie Wilde. 2018. «Hochschulstrategie Als Prozess. Zum Allgemeinen Und Hochschulspezifischen Begriff Der Strategie». https://doi.org/10.5281/ZENODO.1293797.

Meister, Dorothee M., und Gudrun Oevel. 2017. «E-Assessment in der Hochschulpraxis. Empfehlungen zur Verankerung von E-Assessments in der Hochschulpraxis». https://www.dh.nrw/fileadmin/user_upload/e-assessment/pdf/E-Assessment_in_der_Hochschulpraxis.pdf.

Schmees, Markus, und Janine Horn. 2014. E-Assessments an Hochschulen: ein Überblick: Szenarien. Praxis. E-Klausurrecht. Digitale Medien in der Hochschullehre, Band 1. Münster New York: Waxmann. 